\title{
Nuevos aspectos sobre la clasificación de los hongos y su posible aplicación médica
}

\author{
Beatriz Montes ${ }^{1,2}$, Angela Restrepo 2, Juan G. McEwen 2,3 \\ ${ }^{1}$ Corporación Ciencias Básicas Biomédicas, Universidad de Antioquia, Medellín, Colombia. \\ ${ }^{2}$ Corporación para Investigaciones Biológicas, Medellín, Colombia. \\ ${ }^{3}$ Facultad de Medicina, Universidad de Antioquia, Medellín, Colombia.
}

En la última década se ha despertado un gran interés por el estudio de la clasificación de los hongos debido, principalmente, al número creciente de patógenos oportunistas. Actualmente, los métodos tradicionales para la identificación y clasificación de estos microorganismos se basan en criterios morfológicos y en las características de las estructuras de reproducción sexuada y asexuada. En los últimos años, sin embargo, se han demostrado incongruencias de este sistema de clasificación cuando se lo compara con nuevos acercamientos que permiten establecer relaciones de descendencia entre los organismos estudiados. Este artículo revisa varios aspectos novedosos: 1) la ubicación de los hongos en los diversos sistemas de clasificación propuestos en el transcurso de la historia; 2) el reconocimiento de la polifilia de los hongos, gracias al empleo de herramientas comparativas basadas en las secuencias de ácidos nucleicos y en el análisis de las relaciones de descendencia, y 3) los aportes de la filogenética molecular a varios aspectos de interés para la micología médica como son la identificación de los agentes micóticos, su epidemiología y prevención y el diagnóstico de las enfermedades que producen.

Palabras clave: clasificación, hongos, sistemática filogenética.

\section{New fungal classifications and their applications in medicine}

Fungi are gaining importance with the increased incidence of invasive, often fatal mycoses, in immunocompromised patients. In addition, the increased number of emerging opportunistic pathogens has prompted interest in studies pertaining to fungal classification. The traditional methods of identification and classification of these microorganisms are based on the morphology of the sexual and asexual reproductive structures. Recently, this system of classification has been shown incongruent with approaches that better establish phylogenetic relationships among these organisms. Novel aspects of fungal reclassification are described as follows: 1) the position of fungi in the historical classification systems, 2) the recognition of their polyphyly, based on comparative studies of nucleic acid sequences and on analysis of descent lines and, 3) the contribution of molecular phylogeny to medical mycology, including identification of mycotic agents, their molecular epidemiology, prevention of infection and methods of diagnosis.

Key words: classification, fungi, phylogenetic systematics.

En la última década se ha despertado un gran interés por el estudio de la clasificación de los hongos debido, principalmente, al número creciente de nuevos patógenos oportunistas y a

\footnotetext{
Correspondencia:

Juan G. McEwen, Carrera 72ª \#78B-141, Medellín, Colombia.

Teléfono (4) 441 0855; fax (4) 4415514

Beatry7@hotmail.com mcewen@epm.net.co
}

Recibido: 23/12/02; aceptado: 12/05/03 los cambios inesperados que se observan en los patrones de presentación de las infecciones micóticas en el hombre $(1,2)$.

A través de los años, el estudio de la sistemática y de la evolución de los hongos, ha experimentado numerosos cambios (3). Los mayores avances han estado representados por el reconocimiento de la naturaleza artificial del sistema de clasificación e, igualmente, por la polifilia de los organismos tradicionalmente conocidos como 
hongos $(4,5)$. En segundo lugar, está la aceptación de la teoría y de las técnicas de análisis de la sistemática filogenética, así como el desarrollo y aplicación de las pruebas moleculares en el campo de la micología $(6,7)$.

\section{Clasificación de los organismos}

La Tierra está habitada por un número inmenso de seres vivientes; para organizar estas formas de vida de modo que podamos estudiarlas y comunicar apropiadamente nuestros conocimientos acerca de ellas, necesitamos un sistema de clasificación (8).

En el curso de la historia, el hombre ha ideado numerosos sistemas para clasificar las formas vivientes. Generalmente, estos sistemas se catalogan en dos grupos principales: artificial y natural. Un sistema artificial de clasificación se basa en divisiones artificiales o arbitrarias, en las que no hay un reconocimiento de las relaciones entre las diferentes clases de organismos, en el sentido de una descendencia común o relacionada. Por consiguiente, esta clasificación sirve, básicamente, como un sistema de archivo o de registro. En contraste, un sistema natural de clasificación está fundado en las relaciones naturales o evolutivas existentes entre los organismos, reflejando, tanto como sea posible, la probable evolución de estos organismos (9).

\section{Historia de la clasificación}

Los primeros sistemas de clasificación de los seres vivos datan de los tiempos de Aristóteles (300 a 400 A.C.). A partir de entonces se han ideado numerosos y diversos sistemas de clasificación; la mayoría son de tipo artificial y se basan primordialmente en características poco relevantes, lo que explica la controversia existente desde mediados del siglo XVIII sobre las divisiones que habían sido establecidas entre los seres vivos. Lo que comenzó con Carolus Linnaeus como una sencilla división de los seres vivos, en los reinos Animalia y Plantae, se ha convertido en un sistema que se torna cada vez más complejo y sujeto a permanentes modificaciones (8) (cuadro 1).

A mediados del siglo XVIII, Linnaeus ordenó todos los seres vivos en dos reinos bien definidos: el reino Animalia que agrupaba a los animales, organismos móviles y heterótrofos, y el reino Plantae que incluía las plantas, individuos inmóviles, autótrofos y fotosintéticos. Según este esquema, los protozoos quedaron incluidos en el reino Animalia y el resto de los microorganismos, incluso los hongos, en el reino Plantae (10).

Rápidamente, sin embargo, esta clasificación encontró dificultades dado que existían numerosos organismos, como los hongos, que poseían características intermedias que impedían su clara asignación a uno de los dos reinos preestablecidos. Lo único que caracterizaba a los hongos como plantas era su carencia de formas vegetativas móviles. Por su parte, muchas bacterias carecían del poder de fotosíntesis y muchas otras estaban dotadas de movilidad; igualmente, ciertas algas poseían formas móviles que las asemejaban a determinados protozoos; de hecho, algunos flagelados eran estudiados simultáneamente por zoólogos y botánicos (11).

En un intento por resolver el problema, el biólogo alemán Ernst Haeckel propuso en 1866 crear un tercer reino, Protista, también conocido como Protoctista, el que fue reconocido y aceptado. Se incluirían allí todos aquellos organismos con organización biológica sencilla y que fueran unicelulares, cenocíticos o multicelulares, pero que carecieran de especialización tisular, capaces de realizar individualmente las funciones propias y específicas de los tejidos de organismos superiores. Como consecuencia el reino Protista incluyó hongos, bacterias, algas y protozoos (12).

No obstante, la validez del reino Protista fue cuestionada a medida que se obtenía más información sobre la estructura interna de los microorganismos gracias a los avances en microscopía electrónica. Era obvio que estos organismos estaban agrupados por un sistema de clasificación artificial, sin el reconocimiento de las relaciones naturales o evolutivas. Fue entonces cuando, en 1938, Herbert Copeland propuso introducir una modificación al sistema anterior, consistente en remover las bacterias de los protistas, creando para ellas el reino Monera (11).

Posteriormente, en 1969, Whittaker removió los hongos de los protistas colocándolos en el nuevo 
Cuadro 1. Resumen de los sistemas de clasificación en el transcurso de la historia.

\begin{tabular}{|c|c|c|c|c|}
\hline \multicolumn{5}{|c|}{ Historia de la clasificación } \\
\hline $\begin{array}{c}\text { Sistema de dos } \\
\text { reinos } \\
\text { Linnaeus }\end{array}$ & $\begin{array}{c}\text { Sistema de tres } \\
\text { reinos } \\
\text { Haeckel, } 1866\end{array}$ & $\begin{array}{c}\text { Sistema de } \\
\text { cuatro reinos } \\
\text { Copeland,1938 }\end{array}$ & $\begin{array}{c}\text { Sistema de } \\
\text { cinco reinos } \\
\text { Whittaker, } 1969\end{array}$ & $\begin{array}{c}\text { Sistema de } \\
\text { tres dominios } \\
\text { Woese,1990 }\end{array}$ \\
\hline \multirow{4}{*}{ Animalia } & Animalia & Animalia & Animalia & \multirow[b]{2}{*}{ Eukarya } \\
\hline & \multirow{4}{*}{$\begin{array}{c}\text { Protista } \\
\text { (Protoctista) }\end{array}$} & \multirow{3}{*}{ Monera } & \multirow{2}{*}{ Monera } & \\
\hline & & & & \multirow[b]{2}{*}{ Archaea } \\
\hline & & & Fungi & \\
\hline \multirow{2}{*}{ Plantae } & & $\begin{array}{c}\text { Protista } \\
\text { (Protoctista) }\end{array}$ & $\begin{array}{l}\text { Protista } \\
\text { (Protoctista) }\end{array}$ & \multirow[b]{2}{*}{ Bacteria } \\
\hline & Plantae & Plantae & Plantae & \\
\hline
\end{tabular}

reino Fungi. Este investigador intentó colocar los organismos en reinos separados que reflejaran más cercanamente sus supuestas relaciones evolutivas, con base en los dos niveles de organización celular, consistentes en poseer (eucariota) o carecer (procariota) de membrana nuclear. También tuvo en cuenta las tres formas principales de nutrición: fotosíntesis, absorción e ingestión, organizando así a los reinos Monera (procariotes), Protista (eucariotes unicelulares), Plantae (eucariotes fotosintéticos), Animalia (eucariotes fagotrofas) y Fungi (eucariotes osmotrofas). En el esquema de Whittaker, los microorganismos quedaron incluidos en los reinos Monera (bacterias), Protista (protozoos y microalgas) y Fungi (hongos filamentosos y levaduras) (13). Este acercamiento fue un importante intento para desarrollar una clasificación jerárquica que reflejara las relaciones de estos grupos y también para establecer grupos monofiléticos, es decir, un grupo de organismos que contiene el ancestro y todos sus descendientes (14).

El esquema de los cinco reinos de Whittaker ha sido ampliamente aceptado, pero recientemente Carl Woese y colaboradores realizaron una revisión extensa de este esquema. Woese fue el primero en realizar estudios moleculares comparativos de la organización y la estructura de los ácidos ribonucleicos ribosomales (ARNr), y utilizando sus secuencias descubrió que los procariotes (o moneras) incluían dos grupos distintos: las arqueobacterias, que se encuentran en nichos ecológicos caracterizados por condiciones de vida extremas y las eubacterias que se encuentran en cualquier nicho ecológico siendo las formas más habituales. Woese propuso una nueva categoría de mayor rango que el reino y la llamó dominio; propuso un sistema de tres dominios (cuadro 1), que incluye los siguientes: Archaea (arqueobacterias), Bacteria (eubacterias) y Eukarya (eucariotes), cada uno de los cuales contiene dos o más reinos. Por ejemplo, el dominio Eukarya posee los reinos Fungi, Plantae, Animalia y otros que aún no han sido definidos (15). Este sistema de tres dominios se conserva y es el de mayor aceptación en la actualidad.

\section{Reino de los hongos (Fungi)}

Los organismos que pertenecen al linaje de los hongos incluyen setas, royas, trufas, mohos y levaduras, así como otros organismos menos conocidos (14). Se han descrito alrededor de 100.000 especies de hongos, pero se estima que puede existir un número total de 1,5 millones de especies (16).

Los hongos conforman un grupo hermano de los animales y forman parte del superreino o dominio 
Eukarya originado hace cerca de mil millones de años (17). Son un grupo independiente con igual rango que las plantas y los animales; su clasificación se ha basado principalmente en criterios morfológicos y en las características de las estructuras de reproducción (18).

Existen varios grupos de organismos que se cree pertenecen al reino Fungi. Sin embargo, desde hace mucho se han considerado organismos de afinidad incierta (19), debido a que sus relaciones de parentesco aún no son claras y, por consiguiente, se prestan a diferentes interpretaciones (1). En años recientes se han comenzado a aplicar técnicas de biología molecular para intentar una clasificación más natural y lograr así establecer, relaciones más precisas entre estos organismos (7). Aunque ya se dispone de los primeros hallazgos, los resultados de este nuevo acercamiento son aún incompletos puesto que todavía se desconocen muchos aspectos de las interrelaciones entre ellos (20).

\section{Polifilia de los hongos}

Las diferencias de opinión entre los micólogos sobre la clasificación son numerosas y a menudo tan grandes que en la literatura se encuentran discrepancias. Inicialmente, la clasificación general de los hongos partía de dos grandes grupos: 1) Myxomycota, en el que se encontraban los mohos mucilaginosos sin pared celular, y 2) Eumycota, que comprendía los hongos verdaderos con pared (18). En los Myxomycota se agrupaban los filos Acrasiomycota, Hidromyxomycota, Myxomycota, Plasmodiophoromycota. Mientras en los Eumycota se agruparon los filos Mastigomycota (ahora Chytridiomycota), Zygomycota, Ascomycota y Basidiomycota (cuadro 2).

Existen, además, dos grupos convencionales pero que no son reconocidos como grupos taxonómicos formales, a saber, los Deuteromycota (hongos imperfectos) y los líquenes. Los Deuteromycota incluyen todos los hongos que no tienen fase sexuada conocida o que han perdido la habilidad de reproducirse sexualmente. Por tanto, no se sabe con certeza dentro de cuál grupo deberían ser colocados. Los líquenes difieren de
Cuadro 2. Clasificación general de los hongos: divisiones Myxomycota (mohos mucilaginosos sin pared celular) y Eumycota (hongos verdaderos).

\begin{tabular}{|c|c|c|}
\hline \multicolumn{3}{|c|}{ HONGOS (FUNGI) } \\
\hline \multirow{2}{*}{$\begin{array}{l}\text { MYXOMYCOTA } \\
\text { Filos }\end{array}$} & \multicolumn{2}{|c|}{ EUMYCOTA } \\
\hline & Filos & Clases \\
\hline & Mastigomycota & - Chytridiomycetes \\
\hline \multirow[t]{2}{*}{ Acrasiomycota } & & - Oomycetes \\
\hline & Zygomycota & $\begin{array}{l}\text { - Zygomycetes } \\
\text { - Trichomycetes }\end{array}$ \\
\hline Hidromycota & Basidiomycota & $\begin{array}{l}\text { - Teliomycetes } \\
\text { - Hymenomycetes } \\
\text { - Gasteromycetes }\end{array}$ \\
\hline Mixomycota & Ascomycota & $\begin{array}{l}\text { - Hemiascomycetes } \\
\text { - Erotiales }\end{array}$ \\
\hline
\end{tabular}

los otros hongos porque no son un solo organismo, sino más bien una asociación entre un hongo y un alga. Los miembros fúngicos de los líquenes son usualmente un ascomiceto o basidiomiceto, mientras que el alga es usualmente una cianobacteria o una clorofita (alga verde). Con frecuencia, el hongo es incapaz de crecer sin el alga simbionte, lo que dificulta la clasificación de estos organismos (14).

Como se mencionara inicialmente, los hongos abarcan un amplio rango de organismos. Los métodos tradicionales para su identificación y clasificación están basados en criterios morfológicos y en las características de las estructuras de reproducción; estos métodos involucran subjetividad por lo que siempre se ha dudado de la afinidad de algunos de ellos. Los estudios comparativos de las secuencias de ácidos nucleicos (4) han demostrado que los organismos considerados como miembros del reino Fungi no comparten un ancestro común reciente $y$, por tanto, no forman un grupo monofilético en el árbol evolutivo de los eucariotes (figura 1). En este árbol es evidente que algunos organismos estudiados por los micólogos no son realmente hongos, entre ellos los mohos mucilaginosos celulares (Acrasiomycota y Dictyosteliomycota) y el moho mucilaginoso plasmodial (filo Myxomycota); éstos divergen 


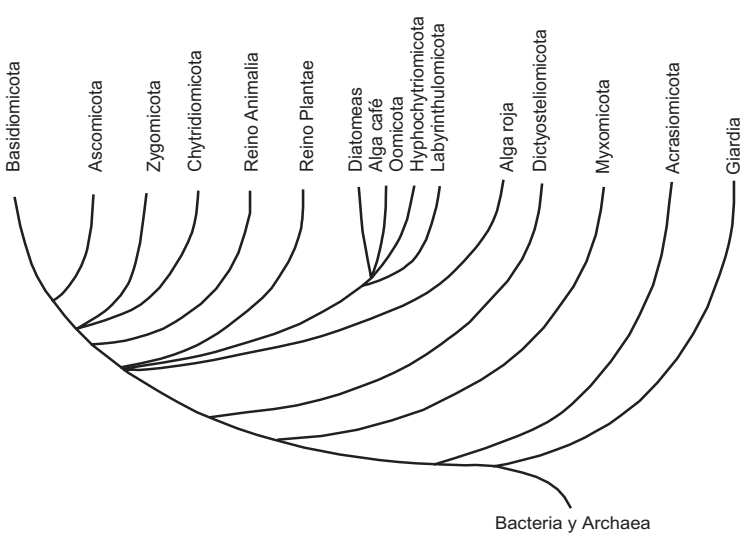

Figura 1. Árbol evolutivo de los eucariotes, construido usando análisis de parsimonia de las secuencias del ADNr 18S. La longitud de las ramas son arbitrarias (2). Reproducida con autorización de Plenum Press, New York, NY.

tempranamente, ubicándose en la región cercana a los miembros del reino Protista como Giardia; por tanto, son clasificados como tres filos de este reino.

Desde hace ya algunos años, los Comycota se han relacionado bioquímica y citológicamente con las algas. La presencia de una estructura tubular tripartita en el flagelo y otros caracteres citológicos han demostrado una estrecha relación entre estos organismos. Esta relación ha sido confirmada por el análisis de las secuencias del ADNr (figura1), que muestra cómo en una misma rama se agrupan algas café, Diatomeas (algas microscópicas), Oomycota y los filos Hyphochytridiomycota y Labyrinthulomycota, formando el reino Stramenopila. Este reino es la adición más reciente al sistema convencional de clasificación y se originó a partir de estudios moleculares que confirmaron que las algas estaban relacionadas con una variedad de protistas; de igual manera, la creación del reino Stramenopila se basa en caracteres morfológicos como lo es la estructura del flagelo (21).

En la parte superior del árbol (figura1), se observan los tres grupos mayores: las plantas, los animales y el reino Fungi. El último está representado por los cuatro filos de los llamados hongos verdaderos: Chytridiomycota, Zygomycota, Ascomycota y Basidiomycota, que forman un clado monofilético (20) y comparten ciertos caracteres con el reino
Animalia como son las estructuras quitinosas, el almacenamiento de glicógeno y el codón UGA para el triptofano. Sólo el filo basal Chytridiomycota posee un caracter primitivo que es un flagelo insertado posteriormente (22).

Los Chytridiomycota han sido caracterizados por poseer células flageladas en su ciclo de vida. Sin embargo, cuando este caracter es utilizado para estudios filogenéticos, se considera un carácter primitivo y, por ello, no se puede usar como criterio para definir a tal linaje monofilético. Los estudios filogenéticos que usan el análisis de las secuencias de ADNr, demuestran que las especies no flageladas colocadas en otros filos pertenecen, en realidad, al filo Chytridiomycota como es el caso de Basidiobulos ranarum (23).

Los Zygomycota, por su parte, han sido delimitados con base en características tales como poseer hifas no septadas y producir zigosporas, pero no se consideran un grupo monofilético. No es clara la posición taxonómica de algunos linajes de micorrizas como los Glomales y los Endogonales, que filogenéticamente pueden estar ubicados fuera de los Zygomycota como linajes basales independientes de los Ascomycota y los Basidiomycota (24).

Los caracteres morfológicos han sido utilizados para dar soporte a las relaciones entre los Basidiomycota y Ascomycota, entre los que se encuentran hifas septadas regularmente y un estado dicarionte (dos núcleos en un solo segmento de hifa). Los resultados obtenidos a partir de la secuencia de ADNr son concordantes y le dan soporte a la hipótesis de las relaciones entre estos dos filos basadas en los datos morfológicos (20).

\section{El concepto de especie en micología}

El concepto más importante en la biología sistemática es el de especie. Es la base de todos los sistemas de clasificación y uno de los conceptos centrales en la biología evolutiva (25); de allí, la importancia de poseer un entendimiento apropiado del término especie. Se trata, básicamente, de la unidad fundamental de la diversidad biológica y, por tanto, aquélla sobre la que actúa la evolución en el proceso que conocemos como especiación $(26,28)$. En general, 
las definiciones de especie se han basado en similitudes fenotípicas, parámetros ecológicos, aislamiento reproductivo, principios evolutivos y combinaciones de los anteriores (29).

Sin embargo, en los hongos existen tres conceptos que han sido objeto de discusión: el concepto morfológico de especie, el concepto biológico y, por último, el concepto filogenético. Las bases genéticas que respaldan algunos de estos conceptos son ampliamente conocidas como sucede con el concepto biológico (capacidad de cruzarse). Sin embargo, la frecuencia de la reproducción asexual en hongos y anastomosis entre las hifas puede causar confusión, ya que en su ambiente natural, el micelio es visto como unidad fisiológica y ecológica cuando, en realidad, es un mosaico genético (30).

\section{Concepto morfológico de especie}

Se basa únicamente en las características morfológicas con especial énfasis en las estructuras de reproducción sexual y asexual (2). Este concepto agrupa a los individuos con base en las similitudes observadas en sus estructuras de reproducción, las que constituyen el criterio principal para determinar la especie. De esta manera, el grado de similitud o disimilitud morfológica entre individuos constituye el criterio primario y decisivo del estatus de especie (27).

Entre los caracteres morfológicos utilizados para la clasificación en categorías mayores, por ejemplo, filo, se usan básicamente algunas características de los esporos sexuales como son la forma y el proceso de producción. La presencia y tipo de septo e, igualmente, la presencia de grapa (clamp) de conexión son otras de las características usadas para este fin. Para los niveles taxonómicos más bajos, como es la especie, se emplean la pigmentación, la forma y la topología de estructuras tales como conidias, células conidiógenas, conidióforos y cuerpos fructíferos. En el género Aspergillus, por ejemplo, es posible identificar aproximadamente 175 especies utilizando las características de la célula conidiógena, del conidióforo y de las conidias (2).

Este ha sido el método clásico de identificación para la mayoría de las especies de hongos, pero este concepto se basa en divisiones artificiales que no se apoyan en el reconocimiento de las relaciones de parentesco entre los individuos (31). Posee, además, un valor limitado porque involucra subjetividad en la definición de especie, ya que se basa exclusivamente en el grado de diferencia que podría variar según el observador.

Existe otra dificultad para la aplicación correcta de este concepto. La existencia de especies que morfológicamente son indistinguibles entre sí o cuya morfología varía fuertemente según el ciclo de vida por el que atraviese, complican aún más su uso. Las especies identificadas bajo este concepto y con base solamente en las diferencias morfológicas, reciben el nombre de especies morfológicas o morfoespecies.

\section{Concepto biológico de especie}

Este sistema, que fuera desarrollado antes del advenimiento del análisis filogenético, hace énfasis en el intercambio genético. Una especie se considera como una población de individuos capaces o potencialmente capaces de cruzarse entre sí, produciendo una progenie fértil, la cual se encuentra aislada del resto de organismos por barreras reproductivas intrínsecas (32).

El concepto biológico de especie ocupa un lugar importante en la micología. Antes de la aparición de este concepto de especie, Shear y Dodge en 1927 mostraron que Monilia sitophila albergaba tres especies que podían ser distinguidas por pruebas de apareamiento. Dichas especies eran: Neurospora crassa, Neurospora sitophila y Neurospora tetrasperma (33). Sin embargo, la aplicación de este concepto a los hongos es complicado.

En efecto, existen dificultades para realizar las pruebas de apareamiento en el laboratorio, sin olvidar que en un número importante de hongos, la fase sexuada es desconocida y que aun en el caso de que ésta se conociera, identificar si el producto de un cruce es fértil o estéril no es un procedimiento que se realice con frecuencia en el laboratorio (34).

También existen dificultades para interpretar apareamientos exitosos de poblaciones que provienen de áreas geográficamente separadas. El significado de estos resultados no es claro ya 
que la prueba de apareamiento es incapaz de distinguir entre el actual flujo de genes y el potencial flujo de genes en la naturaleza (35).

Finalmente, el concepto de especie biológica no se puede aplicar en hongos que no sean cultivables en el laboratorio y aquéllos que no entren en meiosis durante el proceso de reproducción. Sólo se puede aplicar a hongos con reproducción sexual, aunque en hongos asexuales existe la posibilidad teórica del intercambio genético a través de la recombinación mitótica o somática (parasexualismo) (36). La importancia del parasexualismo en la naturaleza es un tema controvertido, ya que se inicia con la fusión de hifas vegetativas (anastomosis) provenientes de diferentes individuos, paso que está limitado por la incompatibilidad vegetativa. De igual manera, la parasexualidad no parece ser capaz de hacer una contribución significativa a la recombinación (37).

\section{Concepto filogenético de especie}

Existen varias versiones del concepto filogenético de especie (29). La versión original de Cracraft en 1983, considera a la especie como el grupo más pequeño de organismos que pueda ser identificado y dentro del cual existe un patrón parental de descendencia y ancestros (38).

Este concepto se enfoca sobre rasgos biológicos o caracteres que son usados para reconstruir la historia evolutiva o filogenia de los organismos. Estos caracteres son rasgos o atributos que pueden ser identificados; habitualmente se utilizan caracteres morfológicos, pero recientemente, los moleculares son particularmente útiles en organismos no cultivables e indistinguibles morfológicamente.

Los caracteres fueron denominados según Willi Hennig, padre de la sistemática, como caracteres ancestrales (o primitivos) y caracteres derivados (o más recientes). El criterio mayor del concepto filogenético es que las especies deben ser unidades monofiléticas que compartan caracteres derivados, también llamados sinapomorfias.

Existen varios métodos para la reconstrucción de las relaciones filogenéticas entre los organismos; entre ellos se encuentran la máxima parsimonia y la máxima probabilidad. En máxima parsimonia, la hipótesis filogenética escogida es aquélla con el menor número de pasos o cambios para el actual estado de los caracteres. En máxima probabilidad, la estimación de las relaciones filogenéticas entre los organismos se realiza a través de la computación de las probabilidades de distribución de los caracteres con base en modelos predeterminados de evolución.

El uso del concepto filogenético de especie ha sido propiciado por los cambios en la sistemática tradicional e introducido, principalmente, por el uso del análisis filogenético y la incorporación de las técnicas de biología molecular (39). Este concepto es particularmente apropiado para el estudio de aquellos hongos en los que no se observan estructuras de reproducción; así mismo, permite la asignación de la posición taxonómica y la identificación de hongos no cultivables, como es el caso de Lacazia loboi, que ha sido clasificado dentro del orden Onygenales con otros hongos dimórficos como Paracoccidioides brasiliensis $(40,41)$. Adicionalmente, el concepto de especie filogenética clasifica los hongos meiospóricos y mitospóricos en un sistema común (36).

Con el concepto filogenético de especie, los individuos son agrupados con bastante precisión pero tiene como dificultad importante la definición del límite de especie. El análisis filogenético de un solo gen puede usarse para agrupar individuos en grupos monofiléticos pero no para colocar un límite a la especie. Por ejemplo, si un gen es polimórfico y tiene dos alelos (A, B), este polimorfismo puede representar una variación entre individuos de una especie; pero si el gen es usado para el reconocimiento de especie filogenética, los individuos que comparten el alelo A pueden ser adscritos a una especie excluyendo a aquéllos que comparten el alelo B (figura 2).

Si se incluye otro tipo de información, como la habilidad para cruzarse, la confusión podría rectificarse. Sin embargo, se ha mencionado ya que muchos hongos no pueden ser sometidos al proceso de cruzamiento y sin esta información adicional no se podría saber si el gen es polimórfico dentro de la especie o está fijado en dos especies diferentes (38). La subjetividad al 


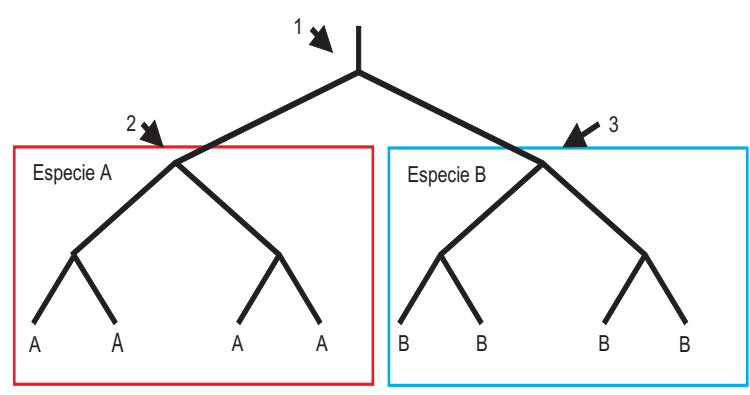

Figura 2. Límite de la especie con la genealogía de un gen. El límite puede estar en la flecha $1 \mathrm{si}$ el polimorfismo es una variación entre individuos de una misma especie. Por el contrario, el límite quedaría en 2 y 3 si los alelos están fijados en especies aisladas genéticamente (38). Reproducida de: Taylor JW, Jacobson DJ, Kroken S, Kasuga T, Geiser D, Hibbett, et al. Phylogenetic species recognition and species concepts in fungi. Fungal Genet Biol 2000;31: 21-32, con permiso de Elsevier Science.

establecer el límite de la especie puede corregirse con el uso de un nuevo acercamiento llamado "concordancia genealógica para el reconocimiento de especie filogenética". Esta metodología se basa en comparar las genealogías de varios genes; cuando los árboles muestran la misma topología (concordancia), se debe a la previa fijación de los loci polimórficos. Por el contrario, la incongruencia en la topología indicaría flujo de genes; la transición de concordancia a conflicto delimitaría la especie (figura 3).

Los estudios filogenéticos de poblaciones de hongos basados en el uso de datos moleculares, han revelado que, realmente, muchas morfoespecies, ampliamente reconocidas en micología clínica como Histoplasma capsulatum, Coccidioides immitis, Aspergillus flavus y Candida dubliniensis comprenden, en realidad, más de una especie filogenética $(42,45)$, las que están provistas de características fenotípicas diferentes tales como patogenicidad e inmunogenicidad $(46,47)$.

\section{Aporte de la filogenética molecular en la epidemiología, prevención y diagnóstico de las micosis}

Es claro el aporte de la filogenética a la taxonomía. El análisis filogenético con el uso de datos moleculares permite identificar la ubicación taxonómica de los hongos cuya fase sexuada se

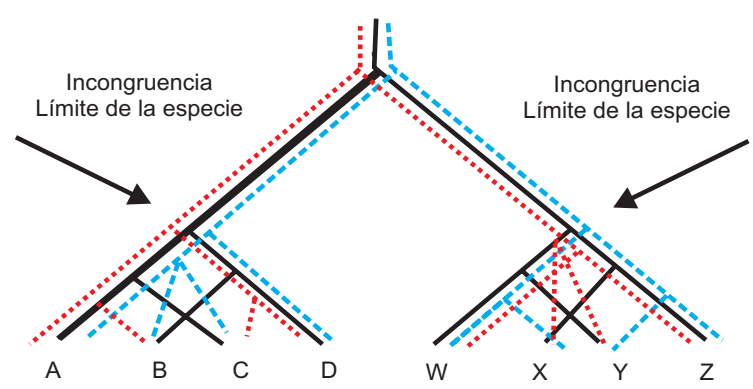

Figura 3. Análisis simultáneo de tres genealogías: se muestra cómo la transición de concordancia a incongruencia entre las ramas puede ser usada para el reconocimiento del límite de la especie (38). Reproducida de: Taylor JW, Jacobson DJ, Kroken S, Kasuga T, Geiser D, Hibbett, et al. Phylogenetic species recognition and species concepts in fungi. Fungal Genet Biol 2000;31: 21-32, con permiso de Elsevier Science.

desconoce y de aquéllos no cultivables como Lacazia loboi microorganismo que, a pesar de los numerosos esfuerzos, no ha sido cultivado ni transmitido exitosamente a animales. Ahora, gracias al análisis filogenético de secuencias del ADNr, se lo ha clasificado dentro del orden Onygenales (41). La filogenética crea igualmente, un sistema común de clasificación para los hongos meiospóricos y mitospóricos (36) y unifica en una metodología (filogenia molecular) la reconstrucción del árbol de la vida (48).

Sin embargo, la taxonomía es sólo uno de los aspectos en el que intervienen los estudios filogenéticos. El reconocimiento de especies aisladas genéticamente tiene un fuerte impacto sobre varios aspectos biológicos aplicados; entre ellos se encuentran los estudios epidemiológicos, la prevención (elaboración de vacunas) y el diagnóstico de las micosis.

El análisis de las poblaciones naturales de hongos está afectado por la capacidad para tipificar molecularmente los aislamientos. Esto se debe a que la tipificación molecular revela un sinnúmero de características de la historia de la vida de estos organismos, las que ayudarían a resolver algunos enigmas sobre la biología y ecología de los hongos. Además, facilita los estudios epidemiológicos al permitir que se establezca la expansión de patógenos humanos (49) y se reconozca si una infección es el resultado de un clon virulento 
o si se debe a la importación de un nuevo aislamiento (50). En el hongo patógeno para el humano, $H$. capsulatum, se identificaron por medio del reconocimiento de especie filogenética, seis grupos aislados genéticamente que fueron correlacionados con características de relevancia biológica tales como distribución geográfica y patogenicidad (46).

Por otro lado, y gracias a los acercamientos comparativos, los estudios filogenéticos contribuyen a predecir algunas características de los organismos basados en las características de sus similares. Es decir, la información se puede transferir a partir de modelos de hongos muy bien estudiados, debido a la facilidad en su manipulación y crecimiento en el laboratorio (como Saccharomyces cerevisiae y Neurospora) a hongos menos estudiados, algunos no cultivables y otros de difícil manipulación (51). La comprensión de las relaciones de los organismos con otras especies es clave para entender sus características, previene las comparaciones impropias basadas en relaciones no existentes e, igualmente, permite identificar características morfológicas o moleculares que explican patrones de comportamientos en la naturaleza.

En el diseño y desarrollo de vacunas son muchos los aspectos que deben ser valorados. EI desarrollo de una vacuna efectiva requiere, además de una adecuada respuesta inmune protectora, determinar el nivel de variación genética en el patógeno (52).

Las especies aisladas genéticamente pueden poseer diferencias en la composición de aminoácidos en las proteínas antigénicas, lo que hace factible la existencia de epítopes con propiedades antigénicas diferentes. La identificación de las variaciones genéticas posibilita la evaluación y elección del mejor candidato para el desarrollo de una vacuna. Es así como en especies morfológicas de hongos de importancia clínica como Coccidioides immitis, que posee más de una especie filogenética $(45,47)$, se encontraron diferencias en la composición de aminoácidos de proteínas antigénicas y, por tal razón, se usan representantes de las dos especies para el desarrollo de una vacuna (53). Las investigaciones en busca de drogas antifúngicas necesitan también del reconocimiento de poblaciones aisladas genéticamente o de clones para estar seguros de que todos los genotipos serán afectados por el tratamiento. De esta manera, el análisis filogenético orienta el desarrollo de programas de control y tratamiento más efectivos.

Los hallazgos de variación en las secuencias de ADN se convertirían en marcadores genéticos para el diseño de nuevas técnicas de diagnóstico de las micosis, las que, a su vez, podrían ser usadas como patrones de comparación entre marcadores moleculares y la relevancia médica de los aislamientos bajo estudio (34). Es así que el análisis filogenético de las variaciones moleculares del genoma de Sporothrix schenckii evidenció la relación existente entre el genotipo de los aislamientos y las formas clínicas de la esporotricosis (54).

En los últimos años, nuestro grupo se ha dedicado al estudio de las relaciones filogenéticas entre aislamientos de $P$. brasiliensis con el uso de caracteres moleculares. El análisis filogenético llevado a cabo con base en la secuencia del exón 2 de la proteína antigénica GP43, ha mostrado un agrupamiento de los aislamientos que se correlaciona con el origen geográfico de los mismos, lo que podría indicar un proceso de especiación por barreras geográficas. Sin embargo, es necesario incluir en este estudio genes adicionales que corroboren esta hipótesis (55).

\section{Conclusión}

El estado actual de los sistemas de clasificación todavía es inestable. Aun los sistemas de clasificación considerados como más modernos y en su mayor parte naturales, son artificiales en algunos aspectos, especialmente en lo que respecta a ciertos campos, como es la micología.

Las herramientas moleculares y los acercamientos a la reconstrucción de las filogenias han aportado una información importante acerca de las relaciones entre los hongos y han permitido la reestructuración del sistema de clasificación. Aunque ya se tienen los primeros hallazgos, los resultados de este nuevo acercamiento son aún incompletos puesto que todavía se desconocen 
muchos aspectos de sus interrelaciones. Sin embargo, el panorama para el uso de la información obtenida a partir de este tipo de estudios es amplio, principalmente, en los estudios epidemiológicos, la identificación clínica de los agentes micóticos y el diseño de nuevas estrategias de prevención.

\section{Agradecimientos}

A John Taylor, Tom Bruns y Martin Bidartondo por su colaboración en la consecución de bibliografia, y a Ricardo Callejas, Myrtha Arango y Alvaro Rúa por su ayuda en la elaboración de este manuscrito. Agradecemos el apoyo a las entidades financiadoras: Fogarty International Research Collaboration Award (FIRCA), proyecto número R03TW01308, Comité de Investigaciones de la Universidad de Antioquia (CODI), proyecto 2044, y la Corporación para Investigaciones Biológicas (CIB).

\section{Referencias}

1. Taylor JW. Molecular phylogenetic classification of fungi. Arch Med Res 1995;26:307-14.

2. Guarro J, Gené J, Stchigel AM. Developments in fungal taxonomy. Clin Microbiol Rev 1999;12:454-500.

3. Redecker D. New views on fungal evolution based on DNA markers and the fossil record. Res Microbiol 2002; 153:125-30.

4. Bruns TD, White TJ, Taylor JW. Fungal molecular systematics. Annu Rev Ecol Syst 1991;22:525-64.

5. Berbee ML. The phylogeny of plant and animal pathogens in the Ascomycota. Physiol Mol Plant Pathol 2001;59:165-87.

6. Wiens JJ. Polymorphism in systematics and comparative biology. Annu Rev Ecol Syst 1999; 30:327-62.

7. McEwen JG, Taylor JW, Carter D, Xu J, Felipe M, Vilgalys $\mathbf{R}$, et al. Molecular typing of pathogenic fungi. Med Mycol 2000;38:189-97.

8. Solomon EP, Berg LR, Martin DW, Villee CA. Diversidad de la vida. En: Solomon EP, Berg LR, Martin DW, Villee CA, editores. Biología de Villee. Cuarta edición. México: Interamericana; 1998. p.479-94.

9. Nason A. Concepto de especie y bases de la clasificación. En: Nason A, editor. Biología. Décima séptima edición. México: Limusa; 1980. p.213-21.

10. Herrera T, Ulloa M. El reino de los hongos o reino Fungi. En: Herrera T, Ulloa M, editores. El reino de los hongos. Micología básica aplicada. México: UNAM; 1990. p.19-24.
11. Audesirk T, Audesirk G. Taxonomía: la imposición del orden en la diversidad. En: Audesirk T, Audesirk G, editores. Biología: la vida en la tierra. Cuarta edición. México: Hall Hispanoamericana; 1996. p.386-97.

12. Corliss JO. Haekel's kingdom Protista and current concepts in systematic protistology. Stapfia 1998;56:85104.

13. Whittaker RH. New concepts of kingdoms of organisms. Science 1969;163:150-61.

14. Alexopoulos CJ, Mims CW, Blackwell M. Systematics of fungal. En: Alexopoulos CJ, Mims CW, Blackwell M, editors. Introductory mycology. Fourth edition. New York: John Wiley \& Sons; 1996. p.61-85.

15. Woese CR, Kandler O, Wheelis ML. Towards a natural system of organisms: proposal for the domains Archaea, Bacteria and Eucaria. Proc Natl Acad Sci USA 1990;87:4576-79.

16. Hawksworth DL. The fungal dimension of biodiversity: magnitude, significance, and conservation. Mycological Research 1991;95:641-55.

17. Baldauf SL, Palmer JD. Animals and fungi are each other's closest relatives: congruent evidence from multiple proteins. Proc Natl Acad Sci USA 1993;90: 11558-62.

18. Arenas R. Taxonomía y clasificación. En: Arenas R, editor. Micología médica. Primera edición. México: Nueva Editorial interamericana; 1993. p.37-41.

19. Alexopulos CJ. Los hongos. En: Alexopulos CJ, editor. Introducción a la micología. Tercera edición. Buenos aires: Eudeba; 1979. p.3-42.

20. Berbee ML, Taylor JW. Fungal phylogeny. En: Oliver R, Scweizer M, editors. Molecular fungal biology. United Kingdom: Cambridge University Press; 1999. p.21-77.

21. Leipe DD, Wainright PO, Gunderson JH, Porter D, Patterson J, Valois F, et al. The stramenopiles from a molecular perspective: 16S-like rRNA sequences from Labyrinthuloides minuta and Cafeteria roenbergensis. Phycologia 1994;33:369-77.

22. Barr DJ. Evolution and kingdoms of organisms from the perspective of a mycologist. Mycologia 1992;84:1-11.

23. Nagama T, Sato $H$, Shimazu M, Sugiyama J. Phylogenetic divergence of the entomophtoralean fungi: evidence from nuclear $18 \mathrm{~S}$ ribosomal RNA gene sequence. Mycologia 1995;87:203-9.

24. Bruns TD, Vilgalys R, Barns SM, Gonzalez D, Hibbett DS, Lane DJ, et al. Evolutionary relationships within the fungi: analysis of nuclear small subunit rRNA sequences. Mol Phylog Evol 1993;1:231-4.

25. Hull DL. The ideal species concept and why we can't get it. En: Claridge MF, Dawah HA, Wilson MR, editors. Species: the units of biodiversity. London: Chapman and Hall; 1997. p.357-80. 
26. Moore-Landercker E. Variation, speciation, and evolution. En: Fundamentals of the fungi. Fourth edition. New Jersey: Prentice-Hall; 1990. p.18-20.

27. Petersen RH, Hughes KW. Species and speciation in mushrooms. Bioscience 1999;49:440-52.

28. Barraclough TG, Nee S. Phylogenetics and speciation. Trends Ecol Evol 2001;16:391-99.

29. Mayden RL. A hierarchy of species concepts: the denouement in the saga of the species problem. En: Claridge MF, Dawah HA, Wilson MR, editors. Species: the units of biodiversity. London: Chapman and Hall; 1997. p.381-424.

30. Kwon-Chung KJ, Bennet JE. Medical mycology. Philadelphia: Lea \& Febiger; 1992. p.3-34.

31. Dettman JR, Harbinski FM, Taylor JW. Ascospore morphology is a poor predictor of the phylogenetic relationships of Neurospora and Gelasinospora. Fungal Genet Biol 2001;34:49-61.

32. Mayr E. Speciation phenomena in birds. Am Naturalist 1940;74:249-78.

33. Shear CL, Dodge BO. Life histories and heterothallism of the red broad-mold fungi of the Monilia sitophila group. J Agric Res 1927;34:1019-42.

34. Perkins DD. How should the interfertility of interspecies crosses be designated? Mycologia 1994;86:758-61.

35. Harrinton TC, Rizzo DM. Defining species in the fungi. En: Worrall JJ, editor. Structure and dynamics of fungal populations. Dordsrecht: Kluwer Press; 1999. p.43-71.

36. Taylor JW, Jacobson DJ, Fisher MC. The evolution of asexual fungi: reproduction, speciation and classification. Annu Rev Phytopathol 1999;37:197-246.

37. Taylor JW, Geiser DM, Burt A, Koufopanou V. The evolutionary biology and population genetics underlying fungal strain typing. Clin Microbiol Rev 1999;12:12646.

38. Taylor JW, Jacobson DJ, Kroken S, Kasuga T, Geiser D, Hibbett, et al. Phylogenetic species recognition and species concepts in fungi. Fungal Genet Biol 2000;31: 21-32.

39. Koufopanou V, Burt A, Szaro T, Taylor JW. Gene genealogies, cryptic species, and molecular evolution in the human pathogen Coccidioides immitis and relatives (Ascomycota, Onygenales). Mol Biol Evol 2001;18:1246-58.

40. San-Blas G, Niño-Vega G, Iturriaga T. Paracoccidioides brasiliensis and paracoccidioidomycosis: molecular approaches to morphogenesis, diagnosis, epidemiology, taxonomy and genetics. Med Mycol 2002; 40:225-42.

41. Herr RA, Tarcha EJ, Taborda PR, Taylor JW, Ajello L, Mendoza L. Phylogenetic analysis of Lacazia loboi places this previously uncharacterized pathogen within the dimorphic Onygenales. J Clin Microbiol 2001;39: 309-14.

42. Sullivan DJ, Westerneng TJ, Haynes KA, Bennett DE, Coleman DC. Candida dubliniensis sp. nov.: phenotypic and molecular characterization of a novel species associated with oral candidosis in HIV-infected individuals. Microbiology 1995;141:1507-21.

43. Franzot SP, Salkin IF, Casedevall A. Cryptococcus neoformans var. grubii: separate varietal status for Cryptococcus neoformans serotype A isolates. J Clin Microbiol 1999;37:838-40.

44. Geiser DM, Pitt JI, Taylor JW. Cryptic speciation and recombination in the aflatoxin-producing fungus Aspergillus flavus. Proc Natl Acad Sci USA 1998;95: 388-93.

45. Koufopanou V, Burt A, Taylor JW. Concordance of gene genealogies reveals reproductive isolation in the pathogenic fungus Coccidioides immitis. Proc Natl Acad Sci USA 1997;94:5478-82.

46. Kasuga T, Taylor JW, White TJ. Phylogenetic relationships of varieties and geographical groups of the human pathogenic fungus Histoplasma capsulatum Darling. J Clin Microbiol 1999;37:653-63.

47. Koufopanou V, Burt A, Taylor WJ. Correction: concordance of gene genealogies reveals reproductive isolation in the pathogenic fungus Coccidioides immitis. Proc Natl Acad Sci USA 1998;95:8414.

48. Doolittle WF. Phylogenetic clasification and the universal tree. Science 1999;284:2124-8.

49. Fisher MC, Koenig GL, White TJ, San-Blas G, Negroni R, Alvarez I, et al. Biogeographic range expansion into South America by Coccidioides immitis mirrors New World patterns of human migration. Proc Natl Acad Sci USA 2001;98:4558-62.

50. Fisher MC, Koenig GL, White TJ, Taylor JW. Pathogenic clones versus environmentally driven population increase: analysis of an epidemic of the human fungal pathogen Coccidioides immitis. J Clin Microbiol 2000;38:807-13.

51. Heidler SA, Radding JA. The AURI gene in Saccharomyces cerevisiae encodes dominant resistance to the antifungal agent aureobasidin $\mathrm{A}$. Antimicrobial Agents Chemother 1995;39:2755-69.

52. Dykhuizen DE, Polin DS, Dunn JJ, Wilske B, PreacMursic V, Dattwyler RJ, et al. Borrelia burgdorferi is clonal: implications for taxonomy and vaccine development. Proc Natl Acad Sci USA 1993;90:101637.

53. Peng T, Orsborn KI, Galgiani JN. Proline-rich vaccine candidate antigen of Coccidioides immitis: conservation among isolates and differential expressión with spherule maturation. J Infect Dis 1999;179:518-21. 
54. Mesa AC, Reyes M, Pérez A, Navarro H, Souza V, Zúñiga $G$, et al. Phenotyping and genotyping of Sporothrix schenkii isolates according to geographic origin and clinical form of sporotrichosis. J. Clin Microbiol 2002;40:3004-11.

\section{Glosario}

Sistemática: organización de conocimientos según determinados conceptos o criterios. Clasificación natural de los seres vivos, basadas principalmente en sus relaciones filogenéticas, utilizando para ello criterios paleontológicos, morfológicos, genéticos, citológicos, embrio-lógicos, bioquímicos, etc.

Sistemática filogenética: un acercamiento a la sistemática que procura recobrar las relaciones filogenéticas (genealógicas) entre grupos de organismos y producir clasificaciones que reflejen exactamente esas relaciones genealógicas. La teoría y la práctica de la sistemática filogenética se definen, así mismo, como filogenia.
55. McEwen JG, Montes BA, García EA, Bagagli E, Restrepo A, Taylor JW. Paracoccidioidomycosis: evolution and strain typing. Annu Rev Biomed Sci 2002 (Special issue): 22.

Taxón: grupo de organismos, animales o vegetales, cualquiera que sea su categoría. Las categorías taxonómicas de uso corriente son: reino, clase, orden, familia, género y especie.

Grupo monofilético: grupo de organismos compuesto por individuos descendientes de un ancestro común.

Polifilia, grupo polifilético: grupo de organismos cuyos miembros descienden de ancestros diferentes. Se considera un grupo filogenética-mente "artificial".

Carácter: atributo o característica de un organismo que puede servir de base para comparaciones con otros organismos. Las diferentes formas de un carácter se conocen como estados del carácter. 